\title{
Técnicas de refuerzo sísmico para la recuperación estructural del patrimonio arquitectónico chileno construido en adobe
}

\section{Seismics retrofiting technics for structural recovery of chilean architectural heritage built with adobe}

\author{
C. Torres Gilles $^{(*)}$, N. Jorquera Silva ${ }^{(*)}$
}

\section{RESUMEN}

El terremoto del Maule del año 2010 de magnitud 8.8Mw, dejó severamente dañadas las estructuras de adobe que conforman gran parte del patrimonio colonial rural chileno. En ese contexto, y como parte de un programa gubernamental de reconstrucción patrimonial, se desarrollaron una serie de intervenciones destinadas a la recuperación estructural y arquitectónica de las obras dañadas, pese a la inexistencia en ese entonces, de una normativa específica que regulara dichas intervenciones. Así, las actuaciones de reconstrucción, refuerzo y consolidación estructural, se realizaron teniendo como referentes investigaciones internacionales, adaptándolas a la realidad de las tipologías locales, en una suerte de laboratorio real donde el desempeño estructural de los inmuebles será puesto a prueba en futuros sismos. En este artículo se caracterizan y analizan comparativamente los sistemas de refuerzo empleados en edificios religiosos y viviendas de adobe, en función de las características tipológicas y de los daños sufridos.

Palabras clave: Refuerzos estructurales, técnicas de intervención, albañilería de adobe, rehabilitación estructural, patrimonio.

\section{ABSTRACT}

The 2010 Maule earthquake of magnitude 8.8Mw, left severely damaged adobe structures that conform the Chilean rural colonial heritage. In that context and as part of a government program of reconstruction, many interventions of structural and architectural restoration of damaged buildings were developed, despite the absence at the time of a specific legislation to regulate such interventions. Thus, the actions of reconstruction, reinforcement and structural consolidation were made taking as reference international researches, adapting them to the reality of Chilean typologies, in a kind of a real laboratory where the structural performance of buildings will be tested in future earthquakes. This article characterizes and analyses comparatively reinforcement systems used in religious buildings and adobe houses, according to their typological characteristics and damages.

Keywords: Structural reinforcements, intervention technics, adobe masonry, structural rehabilitation, heritage.

(*) Académica Facultad de Arquitectura y Urbanismo, Universidad de Chile

Persona de contacto/Corresponding author: claudiatorres@uchilefau.cl (C. Torres Gilles)

ORCID: http://orcid.org/oooo-0002-5577-1385 (C. Torres Gilles); http://orcid.org/oooo-0002-6230-8110

(N. Jorquera Silva)

Cómo citar este artículo/Citation: C. Torres Gilles; N. Jorquera Silva. (2018). Técnicas de refuerzo sísmico para la recuperación estructural del patrimonio arquitectónico chileno construido en adobe. Informes de la Construcción, 70(550): e252. https://doi. org/10.3989/ic.16.128

Copyright: (C) 2018 CSIC. Este es un artículo de acceso abierto distribuido bajo los términos de la licencia de uso y distribución Creative Commons Reconocimiento 4.o Internacional (CC BY 4.0). 


\section{INTRODUCCIÓN}

El terremoto del Maule ocurrido en Febrero del año 2010 de magnitud Mw8.8, ha sido el más destructivo de la zona central de Chile en las últimas décadas, siendo además considerado el quinto evento sísmico más grande registrado a nivel mundial (1). En él, los edificios construidos con estructuras en base a materiales frágiles como el adobe, resultaron ser los más afectados (1). Sin embargo y a pesar de los daños severos, el estado chileno optó por recuperar ese patrimonio, al reconocer que el uso de las albañilerías de adobe forma parte de una rica cultura constructiva local ligada al mundo rural (2) y que las tipologías construidas entre los siglos XVI y XIX con dicho sistema constructivo poseen características muy marcadas (volúmenes de baja altura y grandes cubiertas, recintos herméticos con corredores exteriores) y se encuentran diseminadas en el territorio, otorgando un carácter único al paisaje de los valles del centro de Chile. Este valor patrimonial, se contrapone a la alta vulnerabilidad estructural de dichas construcciones de adobe, debido a su baja resistencia sísmica, sumada a un diseño con muros de gran espesor, sin diafragma que los vincule y una carente mantención, entre otros factores. No obstante, un número significativo de inmuebles con estas características han permanecido en pie luego del terremoto.

Todo lo anterior, puso de manifiesto la importancia de recuperar las obras dañadas, lo que se llevó a cabo mediante programas gubernamentales que permitieron financiar proyectos de intervención estructural en los cuales se buscó prolongar la vida útil de los edificios, recuperando sus sistemas constructivos originales, incrementando las prestaciones de resistencia frente a nuevos eventos sísmicos. Todo ello, con la dificultad en ese momento, de no contar con una norma técnica para intervenir el patrimonio, lo que obligó a hacer cumplir la norma sísmica vigente en Chile, la NCh433/96 (3), diseñada para obras nuevas, en la cual no se consideran los sistemas constructivos con tierra, ya que se estima que dichos tipos de estructuras no son capaces de resistir esfuerzos sísmicos. Por ello, para llevar a cabo los diseños se utilizaron referentes internacionales como publicaciones de relevantes centros de investigación (4), manuales $(5,6)$, y normativas (7), ya que como bien dan cuenta Cid, Mazarron, I. Cañas (8), antes del 2010 existía a nivel internacional una amplia base de normativas publicadas sobre estas técnicas constructivas.

El trabajo de experimentación con intervenciones estructurales, obligó a consensuar criterios de intervención entre autoridades, arquitectos especialistas e ingenieros calculistas, quienes reconociendo la necesidad de recuperar estructuralmente las obras históricas construidas en adobe, promulgaron a fines del año 2013, las normas NCh3332-Estructuras-Intervención de construcciones patrimoniales de tierra cruda-Requisitos del proyecto estructural (9) y la NTM002- Norma Técnica-Proyecto de Intervención Estructural de Construcciones de Tierra (10), las cuales sin embargo, no alcanzaron a ser aplicadas en el gran número de proyectos de intervención realizados en el proceso de reconstrucción patrimonial que acá se mostrarán.

Así, en el presente artículo se busca dar cuenta de los tipos de intervenciones estructurales de mejora del desempeño sísmico de las albañilerías de adobe utilizadas en los proyectos de restauración y reconstrucción en Chile, a partir del levantamiento de información sobre los casos de manera directa en base a visitas en terreno, y de modo indirecto en base a la información publicada de los proyectos. Esto, por medio de un análisis comparativo que contempla la consideración del nivel de daños post-terremoto y las técnicas de intervención propuestas, en dos tipos de proyectos:

- Restauración de Iglesias de adobe, como Monumentos, dentro del Programa de Puesta en Valor del Patrimonio (PPVP)1.

- Reconstrucción de Viviendas de adobe post- terremoto, dentro del Programa de Reconstrucción Patrimonial ${ }^{2}$.

Si bien se trata de dos tipologías distintas, iglesias y viviendas, las obras seleccionadas poseen características constructivas y estructurales en común, por lo que consecuentemente, presen-
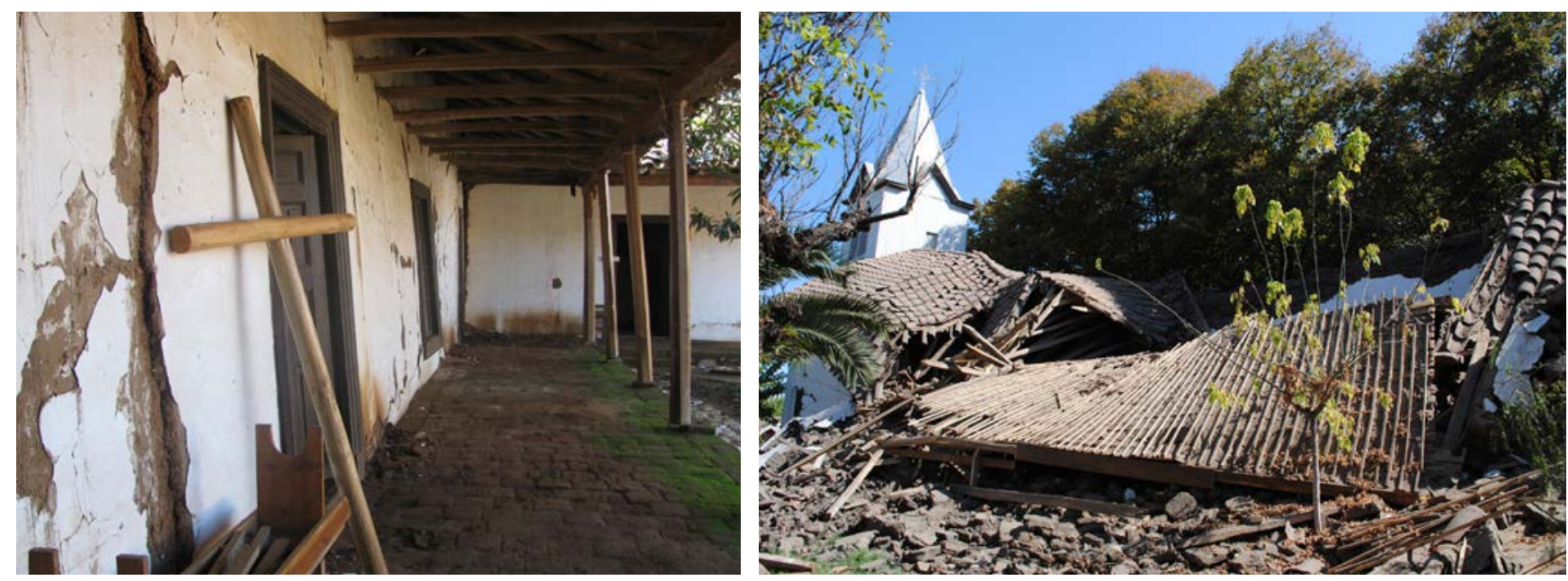

Figura 1. Efectos sismo en Monumentos Nacionales. Izq. Corredor de Casa Parroquial Sagrado Corazón de Jesús de Gualleco. Der. Parroquia Nuestra Señora del Rosario de Guacarhue. Fuente: Claudia Torres y Natalia Jorquera.

\footnotetext{
Programa ejecutado por el Ministerio de Obras Públicas (MOP), iniciado en el año 2008 con financiamiento BID para la restauración de Monumentos Nacionales.

${ }_{2}$ Programa de subvenciones para la reconstrucción post-terremoto 2010, gestionado por el Ministerio de Vivienda y Urbanismo (MINVU).
} 
taron daños similares durante el terremoto del 2010, los cuales responden a patrones de falla característicos del modelo constructivo-estructural de las albañilerías de adobe, tales como grietas de corte en el plano alrededor de vanos, separación de muros en esquinas, volcamientos fuera del plano en tímpanos (4) desaplomes, vaciamientos y colapsos totales o parciales (Fig. 1).

\section{CARACTERIZACIÓN TIPOLÓGICA}

\subsection{Tipologías arquitectónicas}

Los inmuebles estudiados se emplazan en el Valle Central de Chile, zona rural situada entre la cordillera de Los Andes y la Cordillera de la Costa, que se extiende desde la capital Santiago $\left(37^{\circ} \mathrm{Oo} \mathrm{O}^{\prime}, 72^{\circ} 3 \mathrm{O}^{\prime} \mathrm{O}\right)$ hasta la región del Bío-bío $\left(33^{\circ} 27^{\prime} \mathrm{S}\right.$ y $\left.70^{\circ} 40^{\prime} \mathrm{O}\right)$. Es allí donde "se desarrolla una arquitectura de origen español que implantada en el valle central chileno se adapta y asienta, convirtiéndose en algo propio por su completo acuerdo con el clima, topografía, geografía, tradición y técnicas de ese momento" (11), conformada por sencillos volúmenes rectangulares de una única crujía, protegidos por grandes cubiertas de teja donde predomina la horizontalidad, la presencia de corredores exteriores y pocos vanos. "En sus proporciones robustas y escala amplia influyó decididamente el poco costo del terreno y los frecuentes temblores. Ellos explican los enormes espesores de muros y los vanos no muy grandes" (11).

En la mayoría de los casos las viviendas son de tamaño medio (entre 100 y $200 \mathrm{~m}^{2}$ ) y presentan divisiones interiores formando espacios ortogonales de geometría cuadrada. Las circulaciones usualmente son exteriores a través de los corredores que constituyen espacios intermedios, los cuales además "permite(n) la protección del peatón de la lluvia y el sol, a la vez que prolonga la zona de estar de las casas al exterior..." (12). Las viviendas de mayor tamaño cuentan con una circulación interior que cruza por el centro de los recintos, mediante vanos existentes en los muros transversales.

Las iglesias son volúmenes simples de una nave y una sacristía lateral, con torres o campaniles que forman un volumen independiente, muchas veces construido en madera, anexando con posterioridad. Generalmente, a uno de los costados longitudinales, se adosan corredores compartidos con las Casas Parroquiales. Estos inmuebles se posicionan como un gran volumen horizontal en el territorio, acorde a la condición constructiva de la obra.

Como indican Torres y Valdivia (13), "los inmuebles presentan una simbiosis constructiva y unidad de lenguaje arquitectónico entre el espacio de culto, el espacio diseñado para la vivienda y el paisaje rural, entendiéndose los espacios sagrados como una pieza de mayor tamaño dentro del contexto de viviendas de un piso". Por ello presentan sistemas constructivos con características comunes indistintamente de su tamaño y año de construcción.

\subsection{Tipologías estructurales y constructivas}

El sistema constructivo-estructural de estos inmuebles está constituido por muros de albañilería de adobes (bloques de
$30 x 60 x 10 \mathrm{~cm}$ ) levantados sobre cimientos corridos de mampostería de piedras con morteros de cal y tierra. Los muros tienen en su remate superior una solera de madera sobre las cuales se apoya la estructura de techumbre en madera formada por "par y nudillo" o cerchas (en versiones más actuales) (13). En algunos casos, existen también refuerzos horizontales de madera llamados "escalerillas" o "llaves" (Fig. 3), dispuestos entre las hiladas de adobe, los cuales contribuyen a amarrar las albañilerías (2). Este sistema es común en construcciones con tierra de países en zonas sísmicas, ya que estos elementos contribuyen a una mejor conexión entre los paramentos perpendiculares de adobe (14).

La estructura de techumbre soporta la cubierta, generalmente conformada por entablados de madera, una capa de barro y paja que sirve como aislación, y sobre ello se asientan las tejas de arcilla cocida. Los corredores se construyen con la extensión de la cubierta de techumbre apoyada en pilares y vigas de madera (Fig. 1 y 3 )

En la arquitectura residencial, se usa la albañilería de adobes para muros exteriores y la tabiquería de madera rellena con adobe y "adobillos" 3 en los muros de divisiones interiores. También es característico encontrar en las viviendas más antiguas "el uso de los tabiques de quincha ${ }^{4}$ como un aporte indígena previo a la llegada de los colonizadores españoles" (12). Los tabiques, de menor espesor $(20 \mathrm{~cm})$ que los muros, colaboran en la resistencia sísmica de dichas viviendas, absorbiendo los empujes horizontales sobre los muros perimetrales de adobe, resistiendo por lo tanto esfuerzos de corte en el plano. Sin embargo, dado que hay circulaciones interiores entre recintos, estos tabiques presentan vanos que debilitan su rigidez y resistencia estructural. En la arquitectura religiosa en cambio, las naves no cuentan con elementos transversales que ayuden a resistir empujes sísmicos, sólo algunos casos presentan contrafuertes exteriores como productos de alguna intervención de refuerzo posterior a su origen.

Normalmente, los muros de adobe en las viviendas tienen espesores de $60 \mathrm{~cm}$ con alturas que van entre los 3,0m y 4,5m Proporcionalmente, en la arquitectura religiosa estos muros aumentan su altura a 7,om como mínimo, teniendo espesores que van entre los 90 y $120 \mathrm{~cm}$.

\section{TÉCNICAS DE INTERVENCIÓN PARA LA RECUPERACIÓN ESTRUCTURAL}

A nivel internacional existen investigaciones relevantes de propuestas de refuerzo estructural para construcciones en adobe, testeadas en laboratorio y/o modeladas con elementos finitos, realizadas en importantes centros de investigación como la Pontificia Universidad Católica de Lima (15), CRAterre, el Getty Conservation Institute (4) y la Universidad de Aveiro (16), entre otras. En general, dichas propuestas tienen en común el buscar otorgar estabilidad a las obras de adobe, utilizando elementos como mallas o piezas de madera, que restringen el desplazamiento de los muros, previniendo los principales modos de falla y evitando el vaciamiento de los bloques de adobe en los muros, es decir, son propuestas que se preocupan del comportamiento global de la obra, asegu-

\footnotetext{
${ }_{3}^{3}$ Bloque de adobe de menor tamaño (50x15x10cm), que posee una muesca en sus extremos para su ensamblaje con la estructura de madera.

${ }^{4}$ La quincha es un entramado de madera que cuenta con una estructura secundaria a base de elementos vegetales (cañas, bambú, ramas) o de madera de pequeña sección, rellenas con tierra y paja.
} 
rando su estabilidad estructural durante la fase inelástica y el comportamiento último sin colapsos súbitos o frágiles (17). Es por ello que autores como Ortega, Vaconcellos y Correia (18) plantean que el uso de sistemas que permiten incorporar ductilidad al muro como refuerzo, aseguran el buen comportamiento sísmico, práctica que ha sido reportada en otros ejemplos de culturas sísmicas.

Sin embargo, a nivel práctico se plantea reiteradamente el conflicto entre la adecuación de una construcción de adobe para hacerla segura sísmicamente (con la posible destrucción de partes originales de la obra) frente a la opción de preservar la arquitectura original, con el consecuente riesgo de fallas estructurales y colapso total en caso de terremotos (4), abriéndose un debate entre conservación del patrimonio y seguridad de las personas en zonas sísmicas, pues en general "los principios y recomendaciones universales no distinguen la intervención patrimonial entre los casos de monumentos sujetos a la actividad sísmica, y los casos en los que los monumentos no sufren estos embates" (19). No obstante, como un importante referente, la carta internacional de Ravello (1995) indica que "el hecho de escoger entre las técnicas "novedosas o "tradicionales" tiene que ser sopesado caso a caso, y se debe dar preferencia a aquellas que tengan un mayor respeto por el trabajo original y sean menos invasoras" (20), es decir que, los criterios de intervención patrimonial deben adecuarse a cada realidad específica.

En este sentido, es interesante la experiencia chilena luego del terremoto del 2010, pues se plantea como premisa inicial la recuperación del espacio arquitectónico original, en segundo término la seguridad estructural y en tercer lugar la conservación del sistema constructivo tradicional.

Así, en este estudio se analizan las intervenciones realizadas tomando en cuenta las diversas consecuencias del terremoto del 2010, incluyéndose casos extremos de colapso estructural con derrumbes, y casos con daños parciales en que sólo se agrietaron muros y desprendieron revoques (Fig. 1).

Consecuentemente, las actuaciones de intervención se pueden agrupar en tres tipos:

- La "reconstrucción” de la obra, la cual se plantea en casos extremos, cuando los desaplomes de los muros superan el 10\% de su espesor, como deformación máxima admisible (10). Aunque no se haya derrumbado el paramento, se pone en riesgo la seguridad de las personas al quedar vulnerable la estructura frente a nuevos sismos. Un ejemplo de ello es la Casa Parroquial de Gualleco (Monumento Nacional), cuyo pabellón norponiente presentaba un desaplome tras el terremoto de $40 \mathrm{~cm}$. en su punto máximo (en muros de $80 \mathrm{~cm}$ ), es decir se desplazaron un 50\% de su espesor, superando ampliamente el máximo admisible, incluyendo grietas y desprendimientos de revoques (Fig. 1, izq.). Otro ejemplo de ineludible "reconstrucción", fue la Iglesia de Nuestra señora del Rosario de Guacarhue (Monumento Nacional) cuya nave principal colapsó durante el terremoto del 2010 (Fig. 1, der.), pero debido a su gran valor patrimonial, se hizo necesario conservar el espacio arquitectónico.

- Los “refuerzos" según Anexo A de la NCh 433 (3), los cuales buscan modificar las características de una estructura estable dañada o sin daños, de modo que se alcance un nivel de seguridad predeterminado mayor que el original, au- mentando la capacidad resistente de la estructura original. En el caso de las albañilerías de adobes, estos refuerzos se aplican normalmente, para mejorar las prestaciones frente a nuevos empujes símicos.

- En la "consolidación", además de la "reparación" de lesiones menores en que se restituye la capacidad resistente original (3) recuperando las continuidades constructivas de la obra, se busca, dar estabilidad y resistencia a aquellos elementos posiblemente vulnerables frente a futuros esfuerzos sísmicos.

\section{1. "Reconstrucción" con sistemas de refuerzos mixtos}

En todos los casos extremos de "reconstrucción" analizados, se diseñaron sistemas mixtos con elementos estructurales de madera rellenos con albañilerías de adobes. Se utilizan además, fundaciones corridas con sobrecimientos en hormigón armado, de modo que se evite el contacto de los adobes con la humedad proveniente del suelo, asumiéndose las regulaciones normativas en las fundaciones.

Los siguientes sistemas de reconstrucción, sólo se han llevado a cabo en edificaciones con categoría de Monumento Nacional, en este caso, iglesias y parroquias en las cuales se busca la conservación de los sistemas constructivos y la materialidad original de dichos inmuebles, aún cuando éstos ya no ofrezcan prestaciones estructurales. En el caso de viviendas colapsadas en cambio, no se optó por reconstruirlas, sino que mayoritariamente fueron reemplazadas por nuevas construcciones de morfología y materialidades contemporáneas. Los principales sistemas de reconstrucción son:

\section{Albañilería confinada de adobes}

Estas propuestas consideran la construcción de una albañilería tradicional de adobes, resistente a cargas de compresión, confinada entre pilares y vigas de madera, resistentes a flexiones y esfuerzos de corte axial. En este modelo se busca lograr la aprobación de la norma sísmica (3), atribuyendo a las piezas de madera toda la resistencia mecánica, especialmente frente acciones dinámicas.

Mediante los estudios científicos antes mencionados se ha comprobado que "la inserción de elementos de madera dentro de las albañilerías, es un método de refuerzo adecuado, ya que las excelentes propiedades de tracción a lo largo del muro permiten constituir planos de deslizamiento, que tanto vertical como horizontalmente, amortiguan los esfuerzos ayudando a disipar cantidades importantes de energía” (18).

Para poder confinar con madera espesores de muro de $60 \mathrm{~cm}$, se utilizan piezas laminadas o bien se acoplan piezas de madera mediante pernos, sistema usual en la construcción de dinteles. En estos casos se plantea el uso complementario de mallas metálicas de contención en ambas caras de los muros, asegurando la integridad física del paramento.

En casos como el proyecto de restauración de la Casa Parroquial de Gualleco (21), el confinamiento vertical se logra con marcos de madera laminada del mismo espesor de los muros, permitiendo mantener espacios continuos sin elementos sobresalientes. Entre los marcos se disponen piezas de madera (6"x 6") entre las hiladas de adobe, sobre dinteles y como cadena de coronación en el remate superior de los muros 
(Fig. 2). Estas piezas horizontales se unen a los marcos de madera laminada mediante placas metálicas.

Este sistema se cubre posteriormente con revoques de tierra en varias capas, con una primera mezclada con fibra de paja para otorgar mayor resistencia a las tracciones internas en el recubrimiento y evitar que aparezcan nuevas fisuras. No obstante, se asume la posibilidad de aparición de fisuras de menor importancia en futuros eventos sísmicos.

\subsubsection{Albañilería armada de adobes}

En estos casos las albañilerías de adobes se reconstruyen al modo tradicional y se le incorporan elementos estructurales horizontales de madera tipo escalerillas, dispuestos cada 40 5 hiladas (Fig. 3), los cuales permiten la transmisión de empujes sísmicos en el plano y aseguran la continuidad y amarre en las uniones entre muros perpendiculares. Se trata de una especie de albañilería armada, pero con piezas horizontales en vez de verticales.

Tal como indican Ortega, Vaconcellos y Correia, "la introducción de elementos de madera a diferentes niveles de altura en los muros, longitudinal y transversalmente, conecta los elementos entre ellos, amarrando el edificio, potenciando su comportamiento de caja y previniendo su delaminación” (18).

Un ejemplo de este sistema es el aplicado en la reconstrucción de la Iglesia de Guacarhue (22) en la cual además de incorporar nuevos contrafuertes exteriores, se han reconstruido los muros con espesores de $120 \mathrm{~cm}$, en un sistema de albañilería de adobes armados con escalerillas horizontales como refuerzo. Dado el espesor del muro, estas piezas construidas en madera se colocaron cada 3 hiladas.

Para asegurar el sistema, en estos casos también se aplicaron mallas metálicas de contención por ambas caras de los muros.

\subsubsection{Tabiques estructurales}

Los tabiques de madera rellenos (adobes, adobillos o quincha en Chile) tipo Timber Frame han sido históricamente usados en países sísmicos y su comportamiento ha sido ampliamente estudiado. Tal como dan cuenta Poletti, Vasconcelos y Lourenço (23), en estos estudios se determina que, además de las diagonales, la resistencia sísmica de los tabiques estructurales dependerá en gran parte del sistema de unión y el diseño de los conectores entre piezas de madera.
En los casos analizados en Chile, los tabiques se reconstruyen como elementos funcionales arquitectónica y estructuralmente, diseñados como paramentos que dividen los espacios, transmitiendo los esfuerzos perpendiculares a los muros longitudinales y a la vez, ayudando a que éstos vean disminuida la posibilidad de flexionarse producto de los empujes sísmicos, en un rol similar al de los contrafuertes, pero interiores.

Considerando que estos tabiques usualmente tienen vanos de puertas para las circulaciones interiores, se diseñan con entramados de madera de secciones mayores a las originales -de modo que resistan tracciones y compresiones, evitando las deformaciones- y se incorporan diagonales, para que los tabiques sean capaces de resistir los empujes sísmicos en el plano transmitiéndolos al suelo y se logren mayores coeficientes de seguridad. Del mismo modo, es importante que piezas de madera del tabique se inserten en los muros de adobe, de modo que se asegure la correcta colaboración entre muros transversales de distinta materialidad y se eviten lesiones características como grietas verticales en los encuentros de muros perpendiculares (Fig. 4). Los tabiques son rellenados con adobes dispuestos en pandereta o adobillos, de modo que se mantengan como muros macizos y resistentes a cargas de compresión y se conserve la favorable condición de aislamiento térmico entre habitaciones.

Otro de los modos de reconstrucción de los tabiques es mediante un sistema de entablillado de piezas de madera de pequeña sección (tipo quincha) fijos a los pies derechos y rellenos con mortero de tierra y paja aplicado en estado plástico. Para una mejor resistencia a los esfuerzos de corte, el entablillado se coloca de manera diagonal asegurando la transmisión de los empujes horizontales a la base del paramento.

\subsection{Aplicación de refuerzos externos}

Estos sistemas se aplican en obras en las cuales los paramentos han presentado lesiones menores luego del terremoto (en las cuales se mantiene la unidad estructural y el comportamiento mecánico de los muros) o bien, cuando existen lesiones pero reparables. Los elementos aplicados en las intervenciones, buscan reforzar la estructura aumentando la capacidad resistente de los muros, bajo los principios de la mínima intervención y de reversibilidad de las actuaciones.

Los procedimientos realizados corresponden a los indicados en el manual chileno para Evaluaciones de Daños y Soluciones para Construcciones en Tierra Cruda (24), retirándose totalmente el revoque existente (normalmente en malas con-

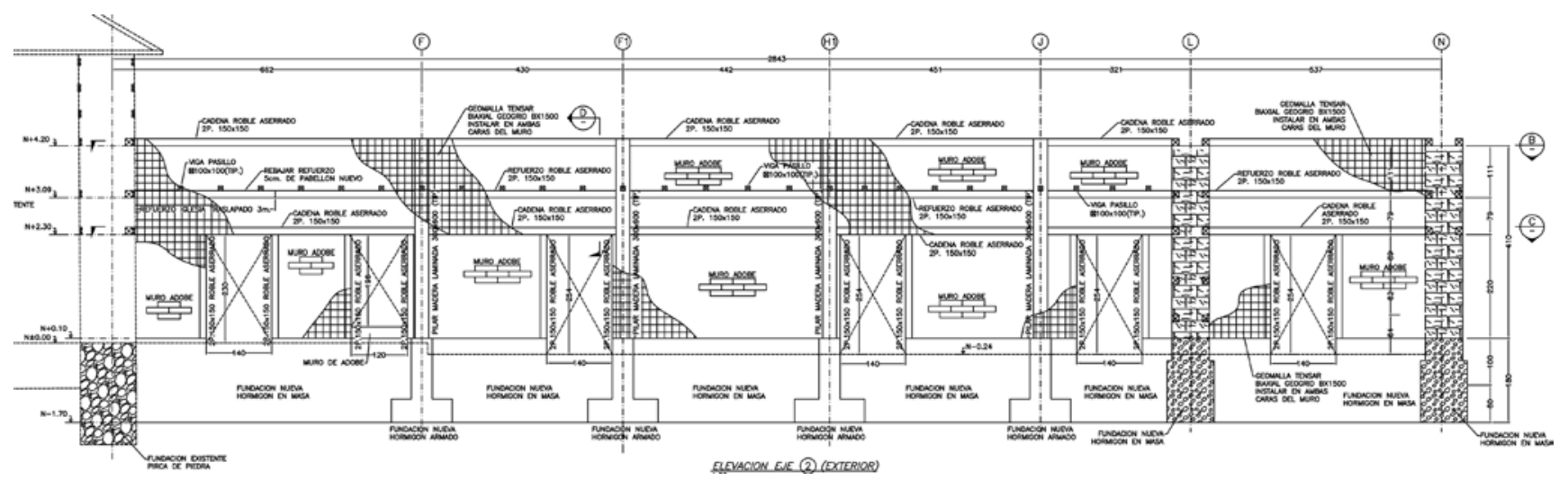

Figura 2. Propuesta de albañilería confinada en Casa Parroquial de Gualleco. Fuente: (21). 

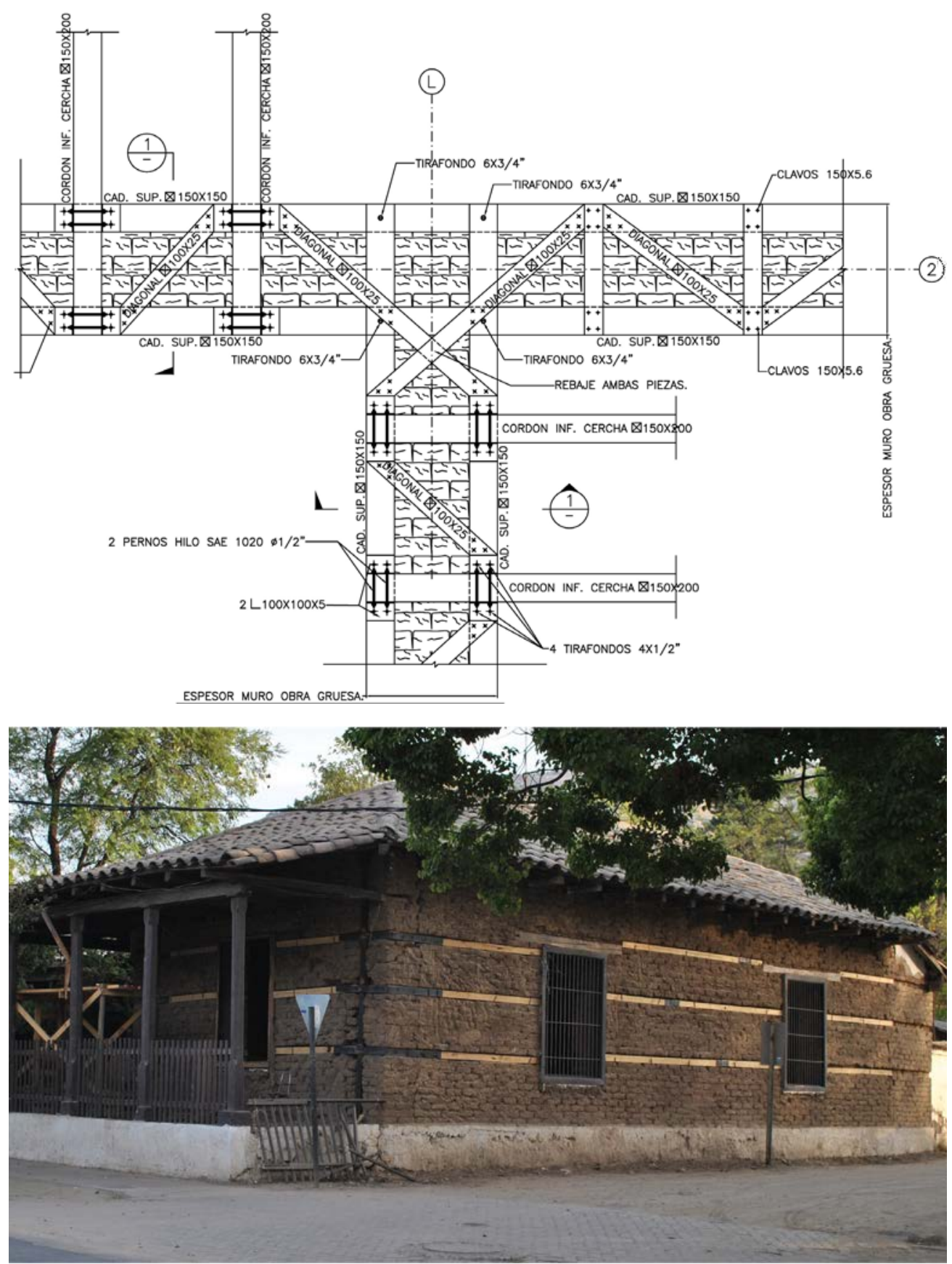

Figura 3. Modelo de escalerillas y propuesta de albañilería armada en una vivienda ubicada en Pichidegua. Fuente: (21) y Natalia Jorquera.

diciones), ejecutándose posibles reparaciones, para luego proceder a la instalación del sistema de refuerzo, reincorporando el nuevo revoque de tierra como terminación.

Los tres sistemas que se presentan a continuación difieren en su materialidad, pero presentan características comunes, como por ejemplo su aplicación por ambas caras de los muros, su amarre transversal y la terminación con revoque de arcilla.

\subsection{Refuerzo con malla electrosoldada}

El refuerzo de muros con mallas metálicas electrosoldadas ha sido estudiado científicamente por ingenieros peruanos $(6$, 15), asegurando que con ellas se "aumenta el nivel de seguridad sísmica de las viviendas de tierra, ya que se incrementa la resistencia de los muros, se controla su desplazamiento y se pospone el colapso" (15). Estos modelos realizados en laboratorios y sometidos a ensayos de resistencia en mesa sísmica han sufrido daños, sin llegar al colapso de la estructura (6). No obstante, los refuerzos de mallas aplicadas en construcciones piloto (6), al igual que los propuestos en algunos manuales (5), han sido concebidos para ser aplicados a viviendas de dimensiones pequeñas, por lo que la aplicación de la malla se limita a los elementos más críticos de la construcción, como encuentro de esquinas y contorno de vanos, mientras en Chile, dada las características constructivas basadas en grandes espesores y altura de los muros, se aconseja aplicar el confinamiento de malla en toda la extensión de los paramentos.

El uso de mallas metálicas electrosoldadas (cuadrícula de $10 \times 10 \mathrm{~cm}, \mathrm{e}=4,2 \mathrm{~mm}$ ) se aplica normalmente para colaborar en la resistencia frente a las tracciones producidas por em- 


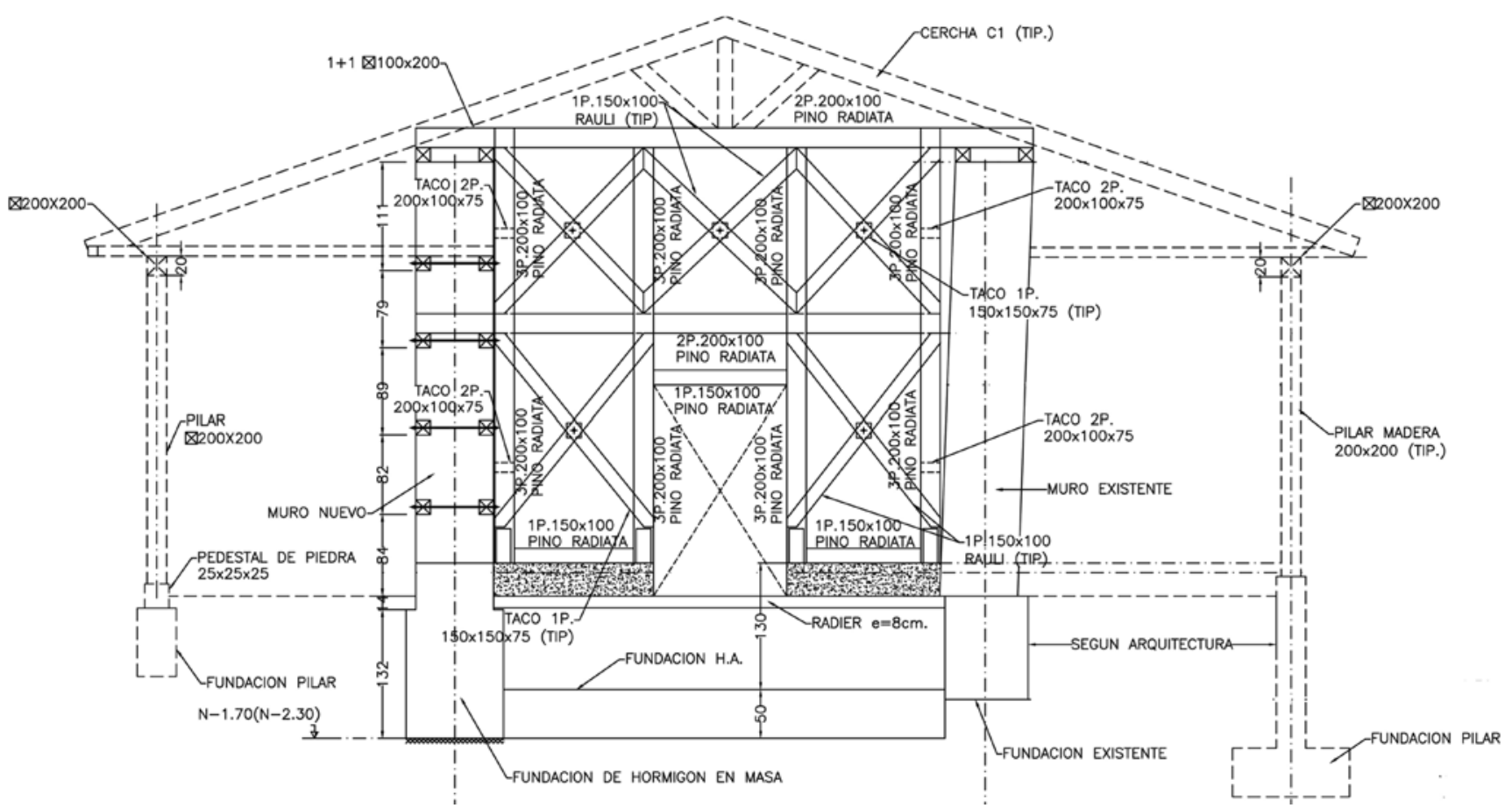

Figura 4. Ejemplo de propuesta de tabique estructural. Fuente: (21).

pujes horizontales perpendiculares al plano del muro, los cuales generan flexiones en los paramentos de albañilerías de adobe. Dado que estos esfuerzos son mayores en la base, se debe asegurar el amarre de la malla metálica a las fundaciones (Fig. 5). Otra función de las mallas, es la contención de los adobes, evitando posibles desprendimientos de piezas o desmoronamientos parciales de muros.

Las mallas se colocan de manera continua envolviendo los paramentos por ambas caras. Se perforan los muros con taladro y brocas especiales -ya que deben traspasar más de $60 \mathrm{~cm}$ - introduciendo en ellos barras de acero que se doblan en los extremos para conectarse a las malla, logrando su amarre. En los vanos de puertas y ventanas la malla se cruza, de modo que la zona que normalmente es más vulnerable a las deformaciones y agrietamientos, quede con un mayor refuerzo y confinamiento.

En algunos casos a la malla se le aplica un antioxidante, en otros se aplica pintura a la cal y en otros, pintura asfáltica para lograr una mayor adherencia con el mortero de recubrimiento. La idea en estos casos es que la malla quede cubierta y protegida de la exposición a la humedad (Fig. 5), para lo cual se revoca con mortero de tierra en varias capas, las primeras con paja y las últimas de arcilla mezclada con cal y arena. $\mathrm{Al}$ amarrarse y recubrirse las mallas con morteros, el muro mejora su desempeño frente a los esfuerzos de corte sísmico en el plano del muro.
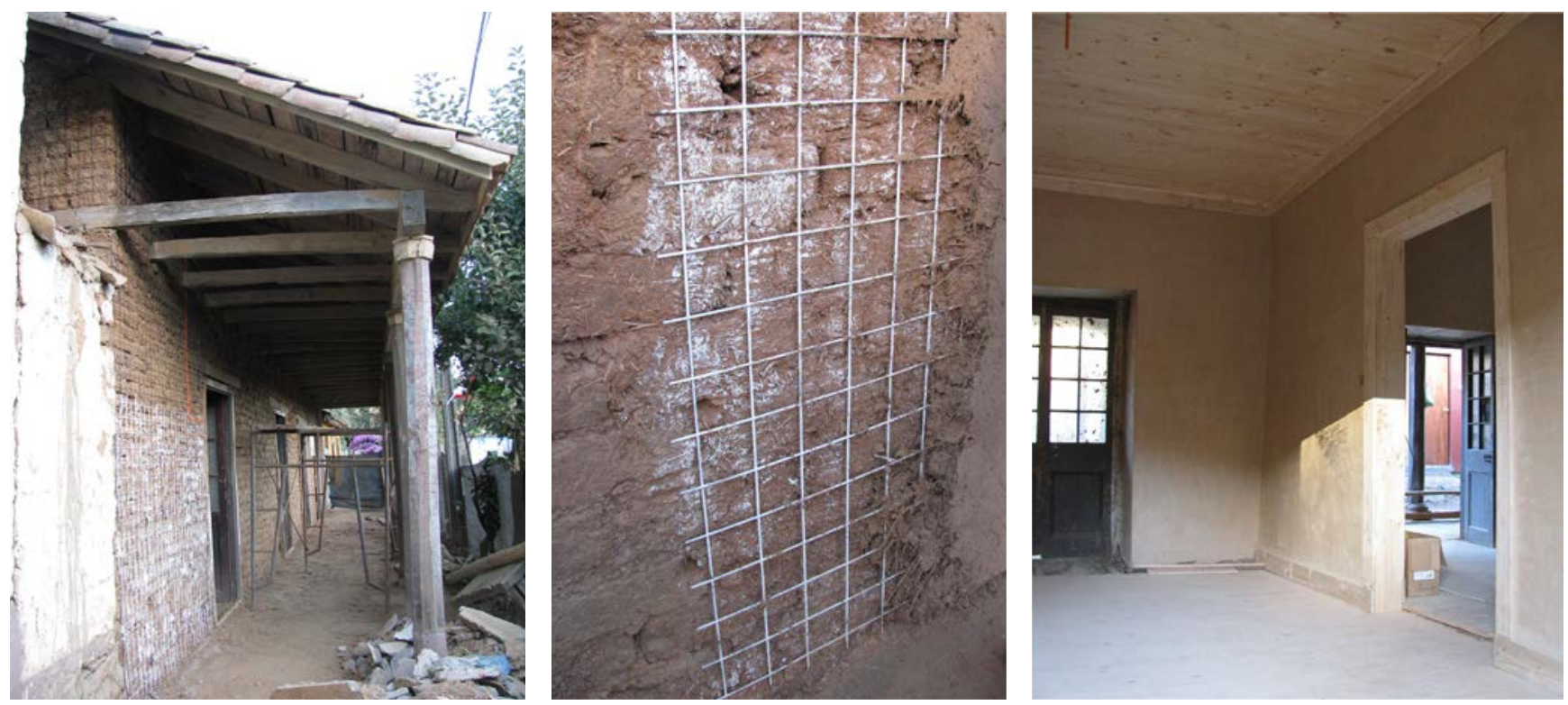

Figura 5. Aplicación de malla electrosoldada como refuerzo en muros de adobe. Recuperación de viviendas en Vichuquén. Fuente: Claudia Torres. 
Cabe mencionar que en Chile, el uso de mallas metálicas lleva unas décadas de aplicación (las malla hexagonales galvanizadas fueron las primeras en ser usadas), usualmente se usaban con morteros de cemento. Sin embargo, en el terremoto del 2010 se demostró que estos recubrimientos de cemento con malla en tabiques de madera (usualmente de róble o pino oregón) fallaron, principalmente, porque las cargas debidas al espesor de los morteros fueron excesivas, pero también debido a la incompatibilidad mecánica de los materiales, si se comparan los módulos de elasticidad dinámicos del hormigón que es de mínimo 18.200 Mpa (25) con el del roble, por ejemplo, que es de 10.700 MPa (26).

Debido a ello actualmente, se usan revoques de tierra, de mejor compatibilidad mecánica con el muro. No obstante, se cuestiona la compatibilidad química del acero de la malla con las arcillas de los revoques, las cuales pueden incidir en el proceso corrosivo del metal y la pérdida de su capacidad resistente.

En general este sistema de refuerzo se ve aplicado con mayor frecuencia en viviendas que en monumentos, esto por el costo relativamente bajo del sistema y la gran disponibilidad de estas mallas metálicas en el mercado local.

\subsubsection{Refuerzo con geomalla}

El refuerzo de muros mediante la utilización de geomallas ${ }^{5}$ tiene como función exclusiva resistir las tracciones inducidas por las solicitaciones sísmicas. Aunque la malla sintética no colabora en entregar una mayor rigidez a la albañilería de adobes, ensayos de laboratorio realizados en la Universidad e Aveiro (15), así como en la PUCP $(6,27)$, indican que, mejora la capacidad de deformación y ductilidad del muro frente a solicitaciones horizontales, además de garantizar su compatibilidad con los materiales originales.

Su aplicación en edificaciones tradicionales en Chile busca asegurar la integridad física de los muros frente a futuros sismos de gran magnitud, aprovechando el buen funciona- miento de éstos en la transmisión de cargas estáticas y principalmente de aquellos que cuentan con piezas de madera $\mathrm{u}$ otros elementos que les han permitido mantenerse en pie frente a anteriores sismos. La utilización de la geomalla asegura la contención de la albañilería de adobes ya que su retícula (existen de dimensiones variables), siempre es de menor separación que la malla electrosoldada por lo tanto, su capacidad de confinar es superior evitando desprendimientos no deseados.

De acuerdo a ensayos llevados a cabo por ingenieros peruanos en conjunto con el Instituto Getty de Conservación (14) o con ingenieros de la Universidad de Sevilla (27), se ha demostrado que los refuerzos de malla plástica embebidos en morteros de barro, mejoran la capacidad de deformación y su ductilidad, la cual puede llegar a aumentar 7 veces, al igual que la resistencia a flexión de los muros que puede aumentar 3 veces (27), permitiendo un mejor control de las fisuras, evitando posibles colapsos.

Sin embargo, este sistema de refuerzo no ha sido probado en laboratorio en muros de espesores superior a $40 \mathrm{~cm}$, por lo tanto, la incidencia en el buen desempeño del conjunto es menor, en casos de muros con mayor masa.

En las obras analizadas, la geomalla se instala con ganchos de anclaje a las fundaciones existentes mediante perforaciones a los cimientos.

La malla envuelve todo el muro por ambas caras, cruzando sobre la viga de remate o solera superior, fijándose a las piezas de madera con grapas. Las mallas se conectan, a través del muro, amarradas por rafias o pita de nylon distanciadas cada $30 \mathrm{~cm}$ o $50 \mathrm{~cm}$ a lo ancho del muro (Fig. 6). Los muros que se conservan, son perforados con taladro para conectar ambas caras con los cabos de nylon. En zona de vanos, al igual que el caso anterior, se realiza el retorno con el cruce de la malla proveniente de ambas caras del muro, de este modo se asegura la estabilidad e indeformabilidad de estas zonas vulnerables.
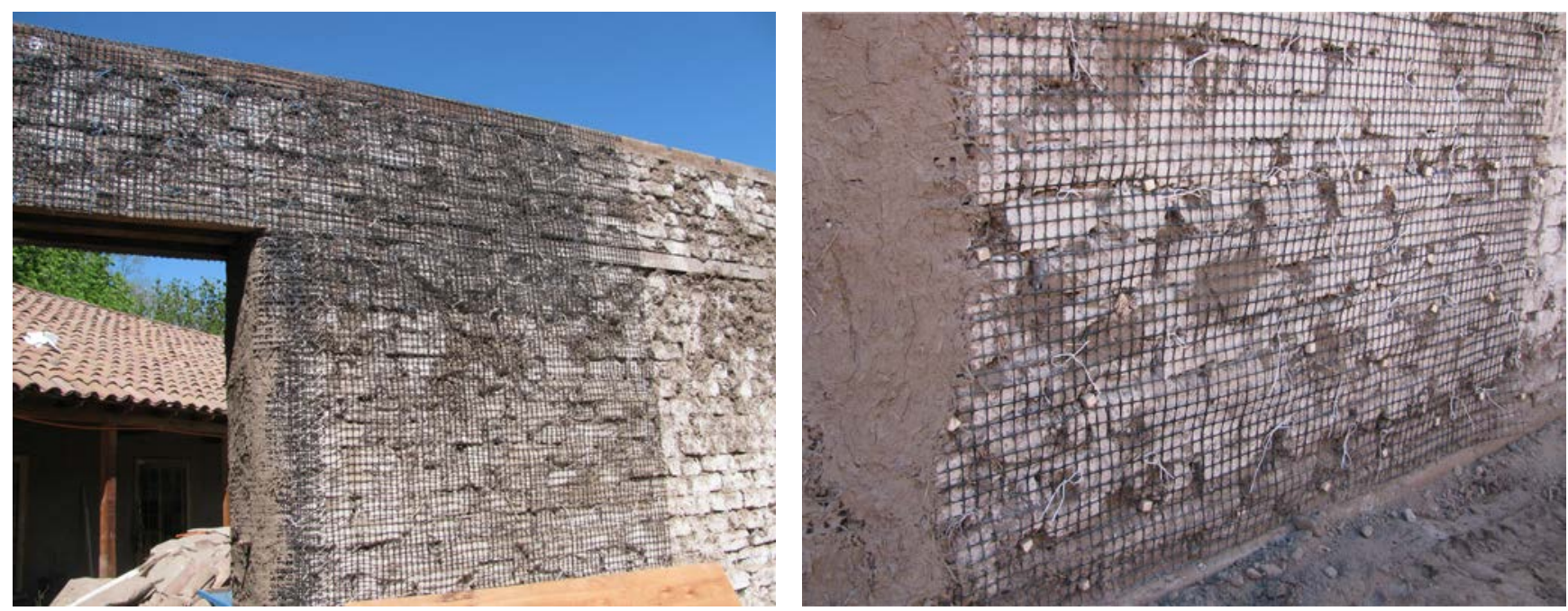

Figura 6. Refuerzo de muros de adobe con geomalla en recuperación de monumentos. Fuente: Claudia Torres.

\footnotetext{
${ }^{5}$ La geomalla es un material en malla de alta tensión (resistencia desde $15 \mathrm{kN} / \mathrm{m}$ para $800 \mathrm{kN} / \mathrm{m}$ ), que puede ser producida utilizando diferentes recursos como fibra (PET, PVA, PEAD y fibra de vidrio) que luego son revestidas con diversos polímeros resistentes al fuego y de alta durabilidad.
} 
La geomalla al estar constituida por polímeros, no necesita una protección especial para la aplicación del revoque ya que su durabilidad es muy alta y no presenta degradaciones en contacto con las arcillas de los adobes o revoques, por lo tanto, se considera altamente compatible con el material de origen. En los casos estudiados se aplica directamente el revoque sobre la geomalla, lográndose una buena adherencia y terminación homogénea.

Este sistema de refuerzo se propone como complemento a los sistemas de reconstrucción, presentados en el punto anterior. Sin embargo, por su alto costo sólo se considera su utilización en edificaciones que tienen protección legal en categoría de Monumentos Nacionales.

\subsection{Consolidación con exoesqueleto de madera}

El uso de exoesqueletos de madera es menos frecuente que el uso de los sistemas de refuerzos mencionados anteriormente. En general es un sistema que se utiliza en muros que han tenido un adecuado comportamiento sísmico, que se encuentran aplomados y que no presentan lesiones graves. Sin embargo, esta consolidación es necesaria ya que estos paramentos, o la unión entre ellos, pueden ser vulnerables frente a sismos de mayor intensidad.

El exoesqueleto, como se indica en (5), es un sistema que integra piezas verticales y horizontales de madera por ambas caras de los muros, otorgando mayor resistencia y rigidez a la albañilería de adobes frente a las posibles flexiones y esfuerzos de corte generadas por los empujes sísmicos. De este modo, la estructura de madera trabaja de modo complementario a la estructura existente (Fig. 7).

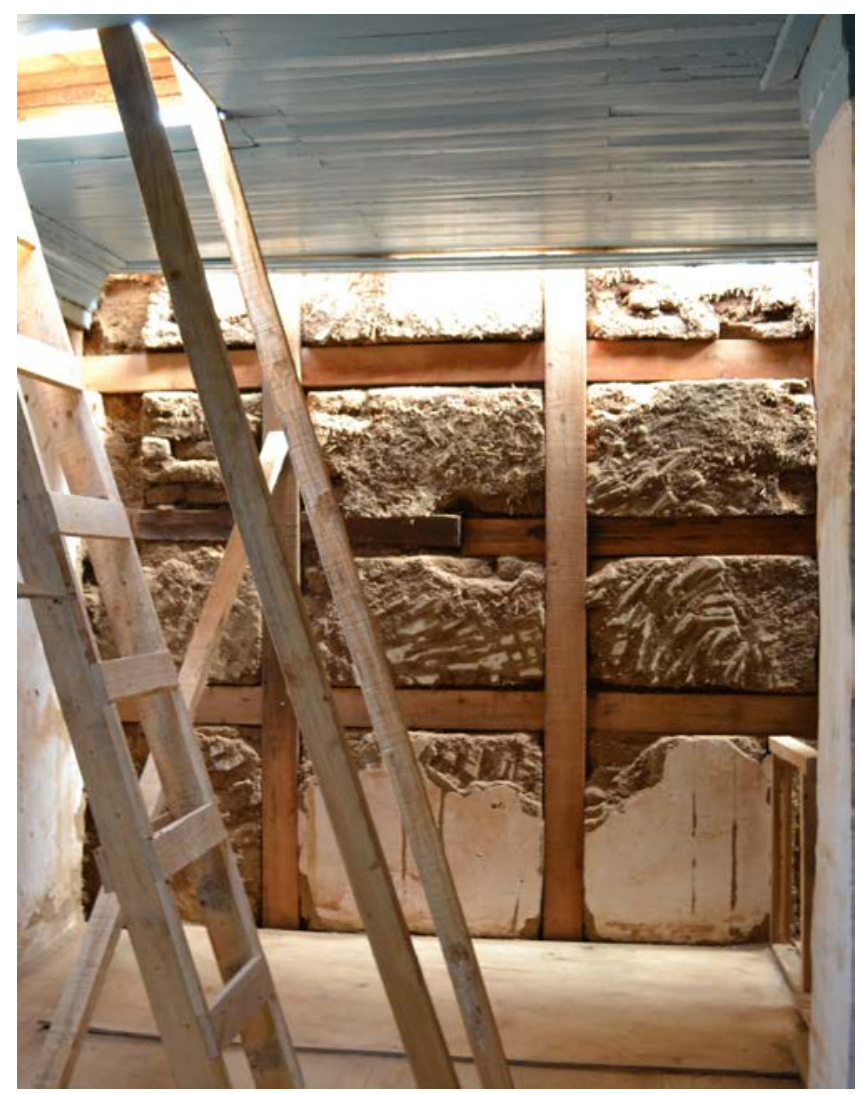

Figura 7. Sistema de consolidación de muros de adobe con exoesqueleto de madera. Fuente: Soledad Valdivia.
En su aplicación como sistema de consolidación, se privilegian los criterios de mínima intervención y de reversibilidad, por lo cual se propone su aplicación parcial en los paramentos. Se plantea el sistema principalmente para consolidar el coronamiento superior de los muros, asegurando las zonas de fijación de la estructura de techumbre, ya que estas zonas superiores son las que por desplazamientos de los muros, frecuentemente presentan desprendimientos o vaciamiento. Así también, se disponen en los contornos de los vanos, ya que los vértices de éstos son los primeros en manifestar las grietas, al ser incapaces de resistir las tensiones que generan las deformaciones producidas por los esfuerzos de corte sísmico. Asimismo, se utiliza como sistema de consolidación y amarre de muros perpendiculares, colaborando con su trabe y de este modo, previniendo posibles falencias existentes en el proceso constructivo de las albañilerías.

La ejecución del sistema se lleva a cabo mediante una retícula de madera $(5 \times 15 \mathrm{~cm})$ dispuestas a distanciadas relativas entre $50 \mathrm{~cm}$ y $100 \mathrm{~cm}$, dependiendo de las distancias entre vanos o muros de apoyo. Estas piezas se instalan por ambas caras de los muros, y se conectan entre sí mediante pasadores metálicos galvanizados.

Para no aumentar demasiado la sección de los muros y asegurar un trabajo mecánico colaborativo entre la albañilería de adobes y las maderas, se hacen rebajes en los muros para insertar al menos las piezas que van en uno de los sentidos y la otra queda de soporte de las capas de revoques de tierra (Fig. 7).

En el uso del exoesqueleto se espera que éste pueda quedar de tope con piezas de madera insertas en los muros, de modo que se fijen mediante clavos o tornillos y así se asegure el trabajo conjunto entre la nueva estructura propuesta y la existente.

\section{CONCLUSIONES}

El carácter extremo o aparentemente invasivo que tuvieron las actuaciones de intervención en las edificaciones patrimoniales en Chile con posterioridad al terremoto del 2010, mediante confinamientos con marcos de madera laminada, armadura con escalerillas horizontales de madera, tabiques estructurales, refuerzos con mallas electrosoldada o geomalla y exoesqueletos de madera, se condice con el amplio espectro de niveles de daño presentado en dichos inmuebles y en ese momento, a la ausencia de una normativa idónea.

Así, en los casos analizados, se pudo apreciar que existe una diversidad de técnicas de intervención que respondieron por una parte, a las diferentes problemáticas que presentaban los casos, como por ejemplo su estado de conservación o diseño arquitectónico original, y por otra parte, intentaban responder a una normativa que obligó a los especialistas a buscar ingeniosamente fundamentar sus propuestas a la sombra de una norma de diseño sísmico que está creada para proyectos de obra nueva.

Si se observan las técnicas propuestas en estos proyectos, podemos ver que por un lado, muchas de ellas se basan o inspiran en prácticas de la propia cultura constructiva del país, dado que en Chile "la experimentación con las técnicas tradicionales ha sido impulsada en gran medida por los frecuentes terremotos, que al destruir parte importante del patrimonio 
arquitectónico, han desafiado su reconstrucción conservando las técnicas ancestrales de construcción, mejorando aquellos aspectos considerados deficitarios en ellas" (28) como por ejemplo la inclusión de piezas de madera en muros de adobes, los distanciamientos máximos entre muros perpendiculares, etc.; y por otro lado, se basan en investigaciones, ensayos, normas, manuales o documentos relacionados a la intervención o construcción en tierra de origen extranjero, principalmente peruanos o colombianos, como una manera de aplicar los conocimientos que en otros países han sido estudiados. Sin embargo, esto implica cierto riesgo ya que las condiciones locales y tipologías constructivas no son las mismas, por los tanto no se puede asegurar con ello que sean infalibles.

Lamentablemente en Chile no se han desarrollado aún estudios científicos respecto a las condiciones de resistencia de las tipologías locales de estructuras de adobe, más allá de caracterizaciones cualitativas de la arquitectura local, ni menos respecto al mejoramiento de resistencia con los refuerzos presentados, de allí la importancia de estos mismos casos con aplicación de distintos sistemas de refuerzo ya que su desempeño será evaluado empíricamente con los futuros sismos. Hasta ahora, estos proyectos han tenido un buen comportamiento mecánico, aunque el tiempo pasado aún es muy breve como para evaluar su resistencia real, por lo tanto, será necesario realizar un monitoreo periódico para su evaluación futura o esperar el próximo gran evento sísmico.

En el estudio se puede concluir además, que la relación entre los diversos métodos de intervención y la valoración patrimonial de la obra, en función de los recursos económicos asignados para su recuperación, demuestran que aquellas intervenciones que responden a criterios más apropiados en cuanto a compatibilidad material y capacidad resistente, en general se aplican a obras de mayor valoración histórica, las cuales cuentan con amplios recursos para su ejecución. En cambio, edificaciones de menor alcance (viviendas) no siempre se ven beneficiadas por intervenciones con materiales adecuados ya que los recursos son restringidos. No obstante, de igual modo se intervienen intentando salvaguardar un patrimonio familiar.

Por último, cabe mencionar que debido a que actualmente existen las mencionadas normativas específicas para la intervención estructural del patrimonio construido en tierra (8-9), las técnicas de intervención realizadas entre el 2010 y el 2013, constituirán un caso único de intervenciones que cumpliendo con la normativa sísmica, debieron dar respuesta masiva a un patrimonio nacional fuertemente dañado.

\section{REFERENCIAS}

(1) Astroza, M., Ruiz, S. and Astroza, R. (2012). Damage Assessment and Seismic Intensity Analysis of the 2010 (Mw 8.8) Maule Earthquake. Earthquake Spectra, 28(1): 145-164.

(2) Jorquera, N. (2014). Culturas constructivas que forman el patrimonio chileno en tierra. AUS (Valdivia), (16):30-35, doi: http://dx.doi.org/10.4206/aus.2014.n16-06

(3) Instituto Nacional de Normalización. (1996). NCh.433.Diseño Sísmico de Edificios. Instituto Nacional de Normalización. Santiago de Chile.

(4) Tolles, E. L.; Kimbro, E.; Ginell, W. (2005). Guías de planeamiento e ingeniería para la estabilización sismorresistente de estructuras históricas de adobe. GCI Scientific Program Reports. Los Angeles, CA: Getty Conservation Institute. http:// hdl.handle.net/10020/gci_pubs/seismic_retrofitting_spanish

(5) Asociación Colombiana de Ingeniería Sísmica. (2005). Manual para la rehabilitación de viviendas construidas en adobe y tapia pisada. AIS. Colombia. http://www.desenredando.org/public/libros/2005/csrvbe/mradobetapia.pdf

(6) Blondet, M.; Villa García, G.; Brezev, S.; et al. (2011). Earthquake-Resistant Construction of Adobe Building: A Tutorial. Earthquake Engineering Research Institute. The World Housing Encyclopedia (WHE). U.S.A. http://www.world-housing.net/wp-content/uploads/2011/o6/Adobe_Tutorial.pdf

(7) Comité Técnico de Normalización de Perú. (2006). Norma E o8o Adobe. Ministerio de Vivienda, Construcción y Saneamiento, Lima, Perú. https://www.sencico.gob.pe/descargar.php?idFile=3478

(8) Cid, J.; Mazarrón, F.; Cañas, I. (2011). Las normativas de construcción con tierra en el mundo, Informes de la Construcción, 63(523):159-169, doi: http://dx.doi.org/10.3989/ic.10.011

(9) Instituto Nacional de Normalización. (2013). NCh.3332. Estructuras-Intervención de construcciones patrimoniales de tierra cruda-Requisitos del proyecto estructural. Instituto Nacional de Normalización, Santiago de Chile.

(10) Ministerio de Vivienda y Urbanismo. (2013). NTM-002. Norma Técnica-Proyecto de Intervención Estructural de Construcciones de Tierra. Ministerio de Vivienda y Urbanismo, Santiago de Chile.

(11) Dávila, R. (1978). Apuntes sobre arquitectura colonial chilena. Facultad de Arquitectura y Urbanismo, Universidad de Chile. Santiago de Chile.

(12) Guarda, G. (1988). Colchagua, arquitectura tradicional. Ediciones Universidad Católica de Chile. Santiago de Chile.

(13) Torres, C., Valdivia, S. (2012). Templos con pies de barro. AS Arquitectura del Sur, 30(41):54-67. http://revistas.ubiobio.cl/index.php/AS/article/view/790.

(14) Jorquera, N. (2014). Culturas sísmicas: Estrategias vernaculares de sismorresistencia del patrimonio arquitectónico chileno. Arquitectura del Sur, 32(46): 6-17.

(15) Blondet, M.; Vargas, J.; Tarque, N.; et. al. (2011). Construcción sismorresistente en tierra: la gran experiencia contemporánea de la Pontificia Universidad Católica del Perú. Informes de la Construcción, 63(523): 41-50.

(16) Varum, H.; Figueiredo, A. \& Silveira, D; et al. (2011). Investigaciones realizadas en la Universidad de Aveiro sobre caracterización mecánica de las construcciones existentes en adobe en Portugal y propuestas de rehabilitación y refuerzo. Resultados alcanzados. Informes de la Construcción, 63(523):127-142.

(17) Vargas, J. (2006). Intervención sismo resistente de estructuras históricas de adobe. En Patrimonio en piedra y adobe: reconstruyendo con la Madre Tierra. Santiago: Cuadernos del Consejo de Monumentos Nacionales(108). 
(18) Ortega, J.; Vasconcelos, G \& Correia, M. (2015). Seismic-resistant building practices resulting from Local Culture. En:Seismic Retrofitting, Learning from Vernacular Architecture. Ed: Correia, M.; Lorenço, P.; Varum, H., London: CRC Press, Taylor \& Francis Group.

(19) Vargas, J., Blondet, M. \& Iwaki, C. (2013). La intervención del patrimonio edificado en tierra en áreas sísmicas y las cartas de conservación. DigitAR (1): 53-61.

(20) Beschaouch, A.; Croci, G. \& Giangreco, E; et al. (1996). Carta de Ravello sobre los aspectos estructurales de la restauración monumental. Loggia, Arquitectura \& Restauración (1): 102-103.

(21) Torres, C., Valdivia S. (2016). Criteria and intervention techniques for seismic resistant recovery of a religious adobe building. En Congreso Euroamericano REHABEND 2016, Patología de la Construcción, Tecnología de la Rehabilitación y Gestión del Patrimonio, Universidad de Burgos, España.

(22) Ministerio de Obras Públicas. (2012). 15 Proyectos de Restauración, Programa de Puesta en Valor del Patrimonio. Ed: Carolina Aguayo, Santiago de Chile.

(23) Poletti, E.; Vasconcelos, G \& Lorenço, P. (2015). Timber frames as an earthquake resisting system in Portugal. En: Seismic Retrofitting, Learning from Vernacular Architecture. London: CRC Press, Taylor \& Francis Group.

(24) Corporación de Desarrollo Tecnológico, CChC. (2012). Evaluaciones de Daños y Soluciones para Construcciones en Tierra Cruda. Manual de Terreno. Documentos Técnicos $N^{\circ}$ 32. Santiago de Chile. Ediciones Raizfutura.

(25) Moravia, W; Gumieri, A \& Vasconcelos, W. (2010). Efficiency Factor and Modulus of Elasticity of Lightweigt Concret with Expanded Clay Aggregate. IBRACON Structures and Materials Journal, 3(2): 195-204. http://www.scielo.br/pdf/ riem/v3n2/05.pdf

(26) Sonderegger, W.; Keunecke, D. \& Baradit, E.; et al. (2010). Selected physical and mechanical properties of the Chilean wood species roble, lingue, manio and alerce. Wood Material Science \& Engineering, Taylor \& Francis Group, 5(1): 5359, doi: http://dx.doi.org/10.1080/17480271003717279

(27) Solís, M.; Torrealva, D. \& Santillana, P.; et al. (2015). Análisis del comportamiento a flexión de muros de adobe reforzado con geomallas. Informes de la Construcción, 67(539):eo92, doi: http://dx.doi.org/10.3989/ic.13.141

(28) Jorquera, N. (2014). Aprendiendo del patrimonio vernáculo: tradición e innovación en el uso de la quincha en la arquitectura chilena. Revista de Arquitectura, 28/29: 4-11. DOI: 10.5354/0719-5427.2014.37087 